\title{
The Platform of Undergraduate Science and Technology Innovation and Entrepreneurship Service
}

\author{
Song Jinbao \\ Information Engineering School \\ Communication University of China, CUC \\ Beijing, China \\ songjinbao@cuc.edu.cn
}

\author{
Wan Jinliang \\ College of Computer and Information Engineering \\ Henan University of Economics and Law \\ Zhengzhou, China \\ wanqq_2006@sohu.com
}

\begin{abstract}
This paper firstly introduces the construction planning, analysis, design and implementation of the undergraduate science and technology innovation and entrepreneurship service platform for the Communication University of China. Secondly, this paper analyzes the new mode of science and technology innovation and entrepreneurship ability training for the undergraduates. At last this paper provides a new way of thinking for talent training, science and technology innovation and entrepreneurship service.
\end{abstract}

Keywords-science and technology innovation; entrepreneurship service; platform construction

\section{INTRODUCTION}

The undergraduate ability of science and technology innovation and entrepreneurship is one of the important contents of China's higher education talent training and an important educational tache to improve the comprehensive quality of the undergraduates. It plays an important role on the cultivation of the student competence, cooperation ability, even the leadership and the ability to make decisions and it is the important means to meet the social demand and promote the undergraduate employment. Constructing science and technology innovation country and science and technology and innovative city has become a national and Beijing's important planning construction goal[1]. The Communication University of China is a famous university in the field of information transmission in China, which has outstanding contribution and the important position in media content and form innovation research, media high technology research and development and the unit decision consultation service for the enterprise or business and so on. It is an important source of the media science and technology innovation. A lot of the media realm student's innovation achievements become productive forces to promote the scientific and technological achievements through guide, cultivating, which will greatly promote the economic growth of the country[2].

\section{THE SIGNIFICANCE OF UNDERGRADUATE SCIENCE AND TECHNOLOGY INNOVATION AND ENTREPRENEURSHIP SERVICE PLATFORM}

The construction of undergraduate science and technology innovation and entrepreneurship service platform is based on science and innovation park of the
Communication University of China, which provides service to the Communication University of China full-time undergraduate and graduate students. The platform is an important part of the modern service industry professional incubation service project and plays an important role in driving the country's science and technology for the whole environmental construction.

How to organize and manage effectively for undergraduate science and technology innovation and entrepreneurship, how to have some pointed guide and cultivation, how to form an organic quality training mechanism together with class education and social education is an important problem which effects undergraduate science and technology innovation and entrepreneurship. And we must put forward the effective solution. The undergraduate science and technology innovation and entrepreneurship services platform construction is to solve these problems, which puts innovation and entrepreneurship quality ability training as the core, is to promote science and technology innovation incubation and achievements of the media as the goal and provides effective information management and service, innovation and entrepreneurship training and guidance, coordination and mutual communication platform for college students with the aid of new media network, modern means of science and technology. The construction and implementation of undergraduate science and technology innovation and entrepreneurship service platform has important significance to improve enthusiasm of undergraduate innovation and enterprise, promote the produce and accelerate conversion of the university students' scientific and technological achievements, improve the success rate of entrepreneurship, regulate the college students' innovative and enterprising organization and management and form the innovation and interactive environment including university students, experts, enterprise and department of government.

\section{THE CONSTRUCTION GOAL OF UNDERGRADUATE SCIENCE AND TECHNOLOGY INNOVATION AND ENTREPRENEURSHIP SERVICE PLATFORM}

The Communication University of China science and technology innovation and entrepreneurship service platform is to support undergraduate innovative and enterprising work as the core target and put public welfare, 
demonstration, profession as the main characteristics. The specific goals are as follow:

1) The $B / S$ interactive network is for foundation platform, combined with the new media, virtualization and other modern means of science and technology[3]. It is for the technical integration and demonstration of information management and service, innovation and entrepreneurship training and guidance, coordination and mutual communication platform construction around undergraduate science and technology innovation and entrepreneurship activities. The Communication University of China undergraduate science and technology innovation activities comprehensive service demonstration system is established including organization, management, training, promotion, resources sharing, the information communication. And the employment and entrepreneurship comprehensive service demonstration system of Communication University of China science and technology park is also set up including entrepreneurship dynamic and hotspots tracking, employment guidance, business training, achievement presentation and investment hatch, project tracking and coordination and communication.

2) The establishment of Communication University of China undergraduate science and technology innovation and entrepreneurship service platform software and hardware test environment and operating system environment will be completed, including undergraduate science and technology innovation and entrepreneurship information service platform internal network, server, video server, cooperation and sharing resources centre server, data information server, user authentication server and browser based client software and security plug-ins, etc[4].

3) Based on the platform, we will assist the Communication University of China and its science and technology park cultivate a group of outstanding college students entrepreneurs, form a coaching professional team, set up a group of college students' successful entrepreneurship model, provide all kinds of enterprise and enterprise project relevant information for college students, organize the business consulting and training in order to improve all kinds of skills of the entrepreneurs and promote enterprise fast growth and independent business[5].

4) Based on the platform, we will effectively extend university science park industry chain, gradually form the "complementary advantages and linkage development" new pattern with other industry inside and outside the park, realize the multiplication effect of entrepreneurship to create more employment opportunities, ease Beijing and radiation regional employment pressure and promote the regional economic growth.

The Communication University of China undergraduate science and technology innovation and entrepreneurship service platform is divided into two parts:

1) Communication University of China science and technology park undergraduate science and technology innovation activities comprehensive service system: a) We will establish a media organization management system of science and technology innovation team, including team building system, the daily operation management system, the reward system, coordination mechanism and the objectives and tasks of the innovative team;

b) We will provide online training and technical guidance environment, focused on training management of the service around university students media science and technology training and innovation technical guidance;

c) We will establish the project results display platform for media science and technology innovation team project intermediary and display needs and provide the innovation and organization management platform for the media project application and competition of national and municipal science and technology innovation;

d) We will establish equipment and experimental environment information management platform and provide the necessary experiment and development conditions for media science and technology innovation team, including equipment and laboratory management, status enquiry, appointment management and necessary training;

e) We will establish a scientific and technological innovation BBS and media team communication space, build teams BBS plate and communication space according to professional direction and form online interactive innovation environment.

2) Communication University of China science and technology park undergraduate entrepreneurship comprehensive service system:

a) We will establish interactive college students' employment information release and online recruitment platform and provide bridge for college students' employment and the enterprises and recruitment direct to innovative team and the outstanding students;

b)We will provide entrepreneurship policy guidance, business dynamic and hot tracking, entrepreneurship case analysis, online entrepreneurship training, science and technology resources service, innovation service navigation and so on focusing on career guidance and training;

c) We will provide results show and hatch investment link and set up the hatch and entrepreneurial tracking information management mechanism according to the needs of the entrepreneurship development;

d) We will create online collaboration and mutual communication platform, implementing online meeting and cooperation project development, white board data synchronization, audio interaction, the resource exchange and sharing function;

e) We will establish a media technology entrepreneurship BBS[6]. 


\section{THE OVERVIEW OF UNDERGRADUATE SCIENCE AND TECHNOLOGY INNOVATION AND ENTREPRENEURSHIP SERVICE PLATFORM}

The Communication University of China undergraduate science and technology innovation and entrepreneurship service platform is divided into two systems. One is undergraduate science and technology innovation activities comprehensive service system. The other is undergraduate entrepreneurship comprehensive service system.

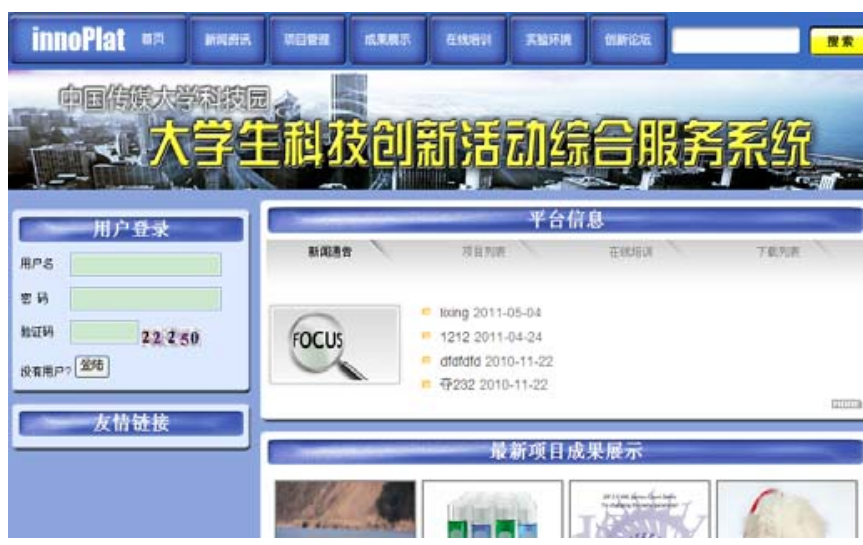

Figure 1. System interface.

\section{A. Science and technology innovation service system}

Fig. 2 is software system frame of science and technology innovation service system. This system is mainly divided into six big modules including user login module, the project application and management module, the innovation achievements exhibit module, online training and technical guidance module, equipment and the experiment environment module and team communication BBS module. The modules specific functions are shown below.

\section{B. Entrepreneurship service system}

Fig. 3 is software system frame of entrepreneurship service system. This system is mainly divided into six big modules including user login module, employment information and online recruitment module, training and policy guidance module, business incubation and entrepreneurial track module, the online collaboration and mutual communication module, team communication BBS module. Among them except employment information and online recruitment module and online collaboration and mutual communication outside module, other modules are similar to innovation service system corresponding modules. The modules specific functions are shown below.

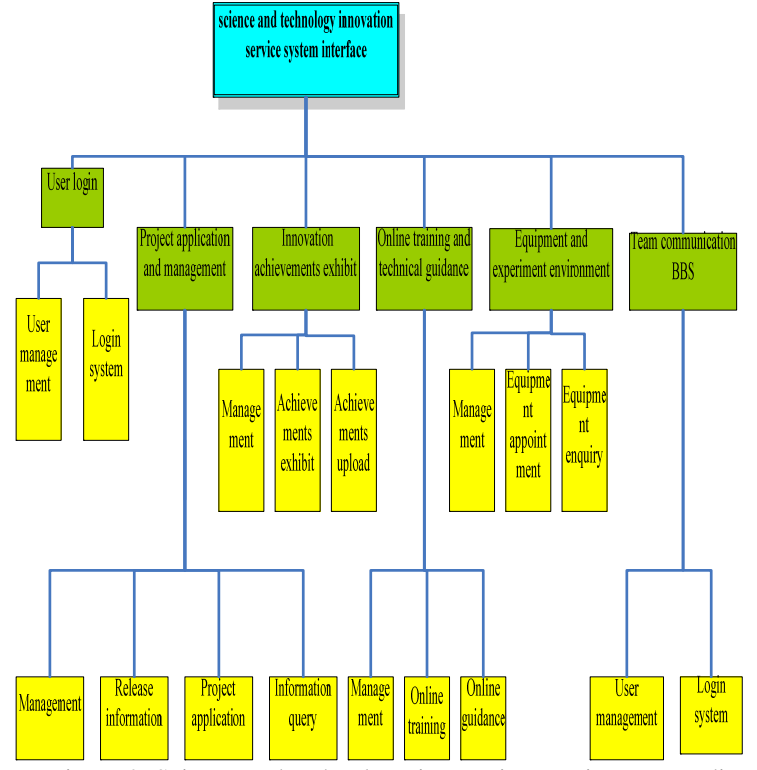

Figure 2. Science and technology innovation service system diagram.

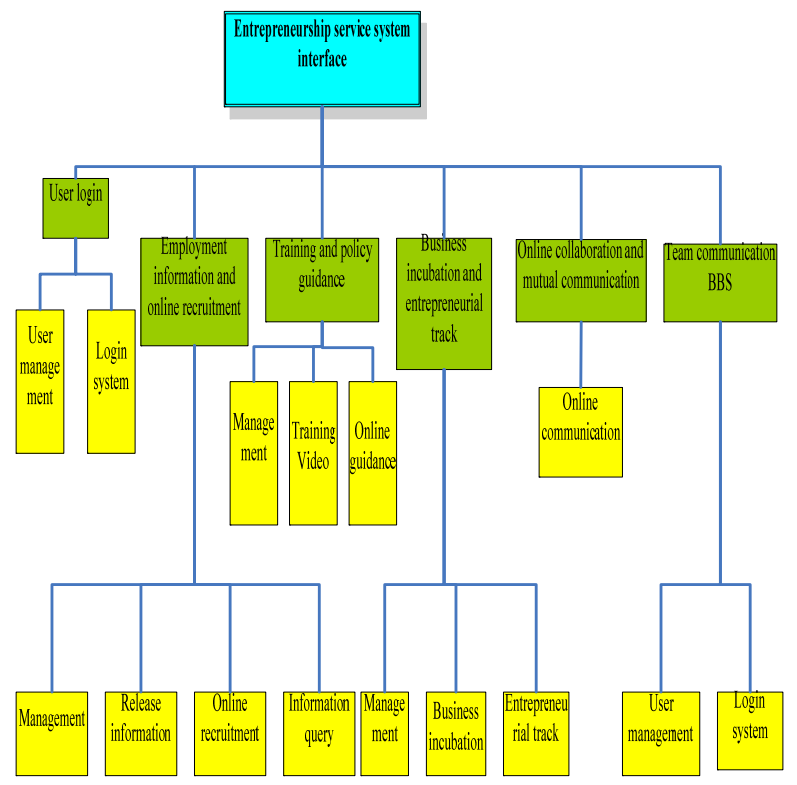

Figure 3. Entrepreneurship service system diagram.

\section{DEVELOPMENT ENVIRONMENT}

A. Software environment summary

- $\quad$ server system: Windows server 2003

- database: mysql 5.1 above

- database graphic interface: navicat more than 9.0

- IDE: myeclipse 8.6 and above

- development of the main language: Java

- JDK version: JDK 1.6 above

- Java web containers: tomcat 6.0 above

- development framework: SSH 
- $\quad$ the browser: firefox or chrome

\section{B. Software environment introduction}

1)JDK: Java SE6 software development kit, is a Java applications foundation. The J2EE is based on Java technology, so installation JDK must be configuration of J2EE environment.

2)Tomcat: Tomcat is a JSP engine developed by Apache organization. It has the function of the Web server, and could be used as independent Web server. But, as a Web server, Tomcat doesn't process static HTML pages more quickly and strongly than Apache. So the Apache and Tomcat is used together by letting the Apache provides processing for the Web static page services and Tomcat JSP engine provides JSP analysis in order to get better performance. Tomcat itself is a sub-project Apache, so that Tomcat provides powerful support to Apache.

3)MySQL: MySQL database system uses the most commonly used database management language-structured query language (SQL) for database management. Using the database graphical interface Navicat can directly add data.

4)Myeclipse: Myeclipse is a free IDE integrated development environment tool which supports the development of language and installing plugins can make its function more powerful.

\section{Struts 2、Spring, Hibernate}

SSH is the abbreviation of Struts2, Spring, Hibernate and is a very popular javaWeb development framework in the current Java world. Also it is a kind of the MVC. MVC is Model, View, Controller layered structure and the three parts should as far as possibly reduce coupling to improve application expansibility and maintainability, shown as Fig. 4. Struts2 is mainly as a control layer, shown as Fig. 5. Spring is mainly used for business layer, shown as Fig. 6. Hibernate is database lasting layer, shown as Fig. 7.

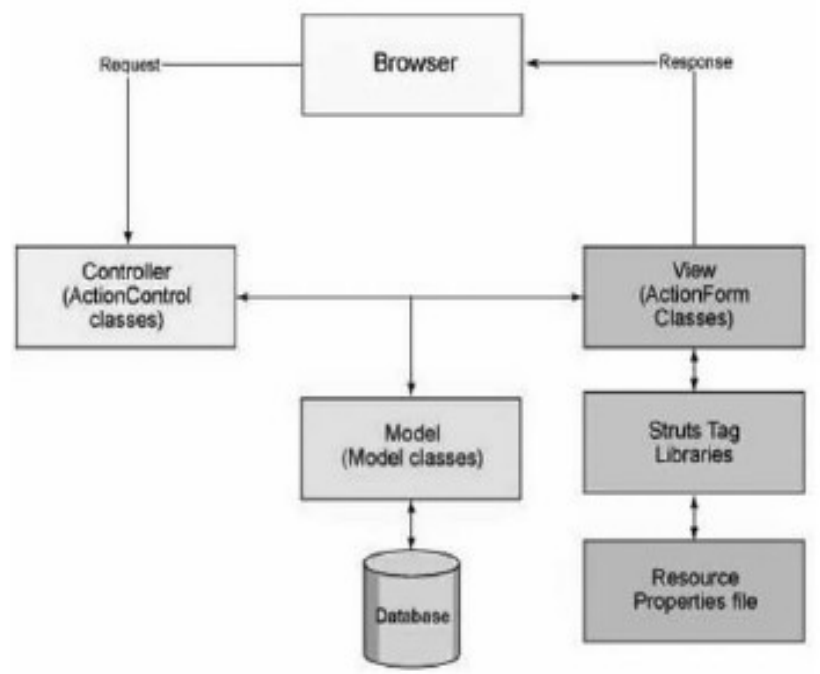

Figure 4. MVC schematic framework.

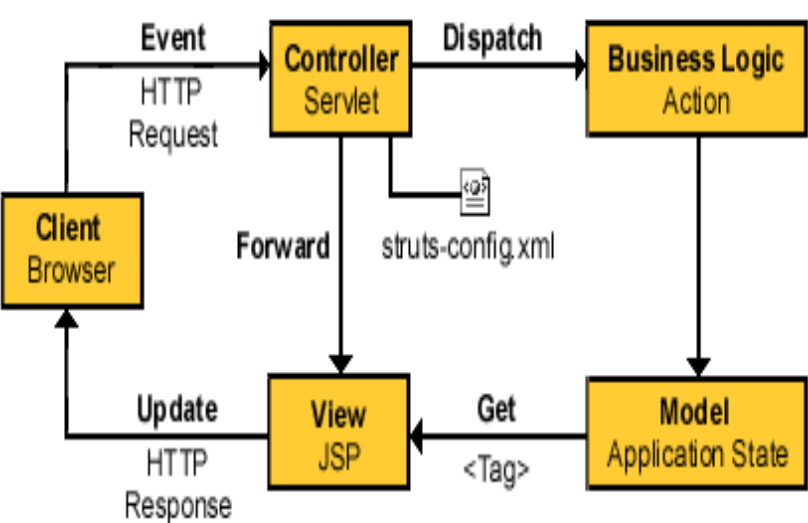

Figure 5. Structs schematic framework.
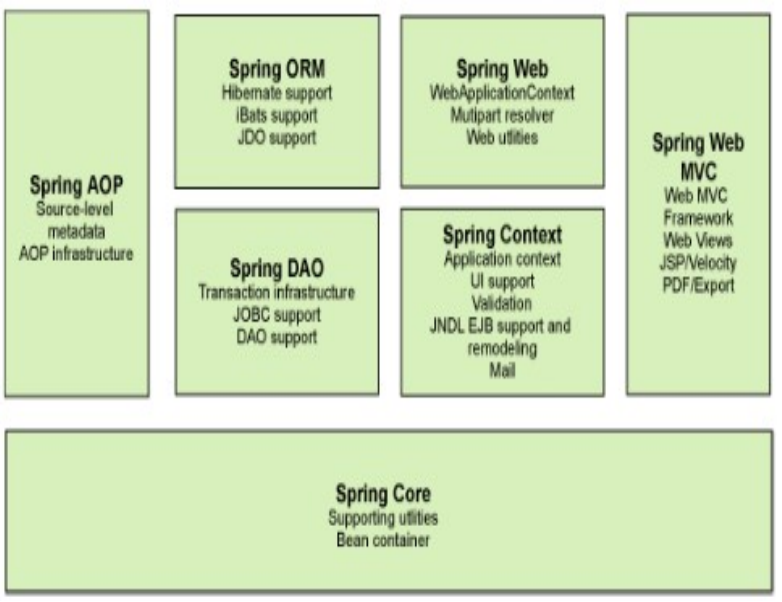

Figure 6. Spring schematic framework.

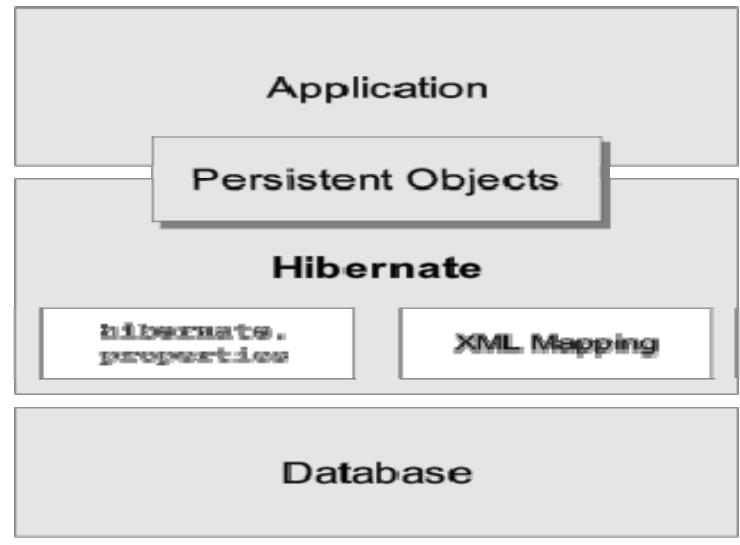

Figure7. Hibernate schematic framework.

\section{SUMMARY}

In the undergraduate science and technology innovation and entrepreneurship services platform construction, we use the technical route of "architecture design" $\rightarrow$ "technical 
model research" $\rightarrow$ "prototype system construction" $\rightarrow$ "demonstration application and perfect system ".

1) We have deeply researched the general framework of undergraduate innovation and business information service platform. In view of the specific requirements of the college students' extracurricular scientific research and science and engineering practice activities, employment, business aspects, and organizational management, innovative and undertaking training, policy guidance, project investment promotion, hatch, equipment resources sharing, online meeting and cooperation, we have done further discussion and demand function analysis, which determines the undergraduate science and technology innovation and entrepreneurship information service platform constraint condition and basic mode.

2) According to the demand analysis and the overall framework of undergraduate science and technology innovation and entrepreneurship information services platform, we have designed the internal structure of the system and researched the Ajax, XMPP, MVC technology[7-9], established technical model that meets demand and architecture requirement, realized the relevant function module, and at the same time synchronously carried on technological verification to ensure the feasibility, reliability and scalability of the technology and the business standard.

3) We have further integrated the software and hardware resources of the undergraduate science and technology innovation and entrepreneurship information service platform, finished a practical integration development, designed and established undergraduate extracurricular technological innovation activities information service demonstration system and undergraduate employment venture information service demonstration system prototype and integrated and tested system to test system function, performance requirements.

4) We have established a practical application of the hardware and software environment. Through actual application verification and testing system at science and innovation park and related universities, we have got through technology difficult points, realized the fusion penetration and general test of the technology, verified the feasibility and reliability of the overall scheme. Also, according to validation and evaluating the effect of the system, we have adjusted and optimized system solutions and perfected the system platform.

\section{ACKNOWLEDGMENT}

Sponsored by Engineering Planning Project of Communication University of China(XNG1230).

\section{REFERENCES}

[1] Song Jinbao. The Communication University of China undergraduate science and technology innovation and entrepreneurship services platform research report, 2012.

[2] Ma Haiyan. The Communication University of China undergraduate science and technology innovation and entrepreneurship services platform work report, 2012

[3] Liu Zhongbing Java laboratory. Java WEB core framework, 2008.

[4] Sun WeiQin. Proficient Hibernate JAVA object persistent mass media technology, 2007.

[5] Rod Johnson, Juergen Hoeller. Expert one-on-one J2EE Development without EJB, 2005.

[6] Christian Bauer,Gavin king. JAVA Persistence with hibernate,2008.

[7] Joseph sack. sql server 2008 transact-sql recipes,2010.

[8] Rod stephens. Beginning database design solutions,2010

[9] Martin Fowler. Patterns of Enterprise Application Architecture,2011. 